\title{
Menschen machen aus Akt und Substanz. Prokreation und Vaterschaft im reproduktions- medizinischen und im literarischen Experiment
}

Caroline Arni

\section{Summary}

As an experimental medical practice artificial insemination in humans dates back to the end of the 18th century. Efforts intensified in the second half of the 19th century, when, especially in France, the number of reports in scientific publications increased and the topic became the subject of heated debates. I trace this emergence of reproductive medicine avant la lettre by reviewing the relevant medical publications. Hereby, I focus on how experiments in artificial insemination presupposed a new conceptualisation of procreation which detaches procreation from the doings of human actors by reducing it to the fusion of germ cells. This "biologisation" of procreation entailed a series of irritations with regard to the determination of "natural" paternity and the impact and relevance of the procreative act's nature. These irritations are dealt with in a novel that appears in Paris in 1884, entitled The Man-Maker. This novel thus attests to the cultural uncertainties that went along with the emergence of what laid the epistemological ground for the reproductive medicine to come.

Keywords: procreation; reproductive medicine; paternity; medicine and literature; 19th-century medicine

\section{Zusammenfassung}

Datieren erste Versuche in künstlicher Befruchtung als humanmedizinische Praxis vom Ende des 18. Jahrhunderts, so griffen seit den 1860er Jahren

Dr. Caroline Arni, Stipendiatin Schweizerischer Nationalfonds, Forschungsstelle für Sozial- und Wirtschaftsgeschichte, Universität Zürich, Rämistrasse 64, CH-8001 Zürich (caroline.arni@ bluewin.ch). 
besonders französische Ärzte das Verfahren vermehrt auf. Bis in die 1880er Jahre entstand eine Serie von Publikationen zum Thema, das schliesslich auch Gegenstand der öffentlichen Diskussion wurde. Der Artikel zeichnet diese Entwicklung anhand von medizinischen Publikationen nach. Dabei befasst er sich mit der Frage, inwiefern diese Experimente eine neue Konzeption von Prokreation voraussetzten, die von menschlichem Tun abstrahiert und Prokreation auf den Vorgang der Keimzellenverschmelzung reduziert. Diese «biologisierte» Konzeption erzeugte indes Irritationen hinsichtlich der Bestimmung von «natürlicher» Vaterschaft sowie hinsichtlich der Bedeutung der Art des prokreativen Aktes. Erschlossen werden diese Irritationen aus dem 1884 in Paris erschienenen Roman Le Faiseur d'Hommes von Yveling RamBaud und Jean-Louis Dubut de Laforest. Dieser Roman dokumentiert die kulturellen Ungewissheiten, welche die Herausbildung der epistemologischen Voraussetzungen der Reproduktionsmedizin des 20. und 21. Jahrhunderts begleiteten.

Dies ist kein medizinischer Roman. Bücher dieser Gattung sind gewöhnlich langweilig und verfehlen das Ziel, das sie sich vornehmen. Sie sind zu gelehrt für den gewöhnlichen Leser, während sie umgekehrt den Ansprüchen des Praktikers nicht genügen. Dies ist kein fantastischer Roman, der auf einer wissenschaftlichen Erkenntnis oder der Variation einer wissenschaftichen Erkenntnis aufbaut [...]. Nein, dieser Roman ist nichts von alledem. [...] Es handelt sich um einen Roman aus dem wirklichen Leben, nicht griesgrämig, sondern ernsthaft, interessant, gesund [...], streng wie die Wissenschaft selbst, die alles läutert, was sie in die Hände nimmt. Dies ist ein ganz modernes Werk, das sich des weiten Feldes der physiologischen Forschung bemächtigt. ${ }^{1}$

Der Roman, von dem hier die Rede ist, erscheint 1884 in Paris und trägt den Titel Le Faiseur d'Hommes. Tatsächlich ist es kein Buch, das die Leserin mit übertriebener Gelehrsamkeit belästigt, und es ist auch kein fantastischer Roman. Zwar spielt der Titel mit dem im 19. Jahrhundert in naturwissenschaftlichen Begriffen erneuerten Projekt des «man-made man»², ruft er doch dystopische Visionen des Fabrizierens von Menschen in dunklen, Funken schlagenden und von grössenwahnsinnigen Wissenschaftlern bevöl-

1 RamBaud/Dubut de Laforest 1884, viif. Dieses und alle nachfolgenden Zitate aus dem Französischen gebe ich in eigener Übersetzung wieder.

2 Tanner 2005, 44. 
kerten Labors auf. Doch erzählt wird nicht von durch Wissenschaftlerhand missraten ins Leben gekommenen Monstern und venusgleichen Automatenfrauen. Der Roman handelt vielmehr von künstlicher Befruchtung, fécondation oder génération artificielle. Und das war 1884 keine Fantasie, sondern eine relativ simple medizinische Intervention, mit der seit Ende des 18. Jahrhunderts zunächst bei Tieren und vereinzelt auch beim Menschen experimentiert worden war und die seit den 1860er Jahren besonders in Frankreich zunehmend von Ärzten praktiziert und kommentiert und schliesslich auch Gegenstand der öffentlichen Diskussion wurde ${ }^{3}$.

Diese «grosse Debatte um die künstliche Befruchtung [...], die zur Stunde die denkende Welt so sehr umtreibt», hätten die Autoren des Faiseur d'Hommes, Yveling RamBaud und Jean-Louis Dubut de Laforest, als erste in der literarischen Welt aufgegriffen, so schreibt der Wissenschaftsjournalist Georges Barral, der auch als Verfasser des Vorworts firmiert, dem obiges Epigraph entnommen ist. Dabei hätten sie «eine Schneise in das Getümmel aus Heuchlern und Dummköpfen» geschlagen und «der Physiologie, wie sie von Claude Bernard entworfen worden ist, ein Asylrecht in der Gegenwartsliteratur» eingeräumt. Deshalb sei der Roman, so Barral, ein Jahr nach dessen Erscheinen, als eines der «gegenwärtig einflussreichsten» literarischen Werke anzusehen ${ }^{4}$.

Indes ist der Faiseur d'Hommes mehr als Zuflucht für biologisches Wissen über Prokreation und eine umstrittene humanmedizinische Praktik ${ }^{5}$. Wohl argumentiert der Roman mit den lebenswissenschaftlichen Fakten, die Barral in seinem Vorwort der Erzählung voranstellt ${ }^{6}$. Doch er entlässt keine

3 Zur Geschichte der künstlichen Befruchtung in Frankreich existieren einzelne medizinhistorische Arbeiten sowie Hinweise in thematisch umfassenderen Studien, sie ist aber noch kaum als eigenständiger Gegenstand und aus wissenschaftshistorischer und kulturgeschichtlicher Perspektive aufgearbeitet. Vgl.: Darmon 1981; David 1987; Gélis 1988,262-265; Gonzalès 1996, 278-282; da Silva 1991; Griset 1995; Rostand 1948, 12ff.; Stora 1976. Vgl. zur Geschichte der künstlichen Befruchtung allgemein sowie im deutschen Sprachraum: Schreiber 2007, bes. 53-118; Benninghaus 2005; Horn 1997; Semke 1996; Hommel 1994; Poynter 1968 (Poynter geht auch auf Frankreich und den hier behandelten Joseph Gérard ein). Vgl. zum Faiseur d'Hommes, allerdings nicht hinsichtlich der künstlichen Befruchtung: Cryle 2004.

4 Barral 1885a, 337. Hinter dem Pseudonym Yveling RamBaud verbirgt sich der Romancier und Dramatiker Frédéric Gilbert (1840-1899); Jean-Louis Dubut de Laforest (1853-1902) war Journalist und Schriftsteller.

5 Der hier gewählte Begriff der «Prokreation» hat den Vorteil, dass er - anders als die Begriffe «Zeugung», «Reproduktion», «Fortpflanzung» oder «Generation»- kein historisch und disziplinär spezifisches Konzept dieser Vorgänge transportiert. Mit Saskia Walentowitz (2007, 37) verwende ich «Prokreation» als einen konzeptuellen Begriff, der die immer historisch und kulturell spezifisch gedeutete und in soziale Fakten übersetzte biologische Erzeugung neuer Menschen meint und dabei die Frage nach diesen spezifischen Deutungen und Übersetzungen als empirische Frage offenhält.

6 Die Erläuterungen in Barrals Vorwort sind teilweise fast wörtliche Übernahmen aus einem Lexikonartikel des Histologen Charles Robin (vgl. Robin 1878). 
Gewissheiten in die Leserschaft, und er ist auch nicht das eindeutige Plädoyer für die künstliche Befruchtung, das er sein will. Wenn auch mit beschränkten literarischen Mitteln, so durchaus in Emile Zolas Sinn, arbeiten die Autoren vielmehr in der Werkstätte des Roman expérimental: Wo der Faiseur d'Hommes das medizinische Experiment der künstlichen Befruchtung in Szene und damit in den Gang nicht nur organischer Phänomene setzt, wird er selbst zu einem literarischen Experiment. Dieser Versuch dokumentiert den Zusammenstoss von humanbiologischem Wissen über reproduktive Vorgänge und einer tradierten kulturellen Konzeption von Prokreation. An der reproduktionsmedizinischen Intervention nämlich war nicht nur brisant, dass der «natürliche» Akt durch einen «künstlichen» Eingriff, Sexualität durch Technik ersetzt wurde. Brisant war, grundlegender, der im medizinischen Experiment erbrachte Nachweis, dass Prokreation nicht - wie bis anhin - als gemeinsames Tun der Eltern, sondern - neu - als ein biologisches Ereignis zu verstehen sei, in dem nicht Menschen handeln, sondern körperliche Substanzen interagieren.

Daraus ergab sich eine gewichtige Komplikation. Als die ärztliche techné die im sexuellen Akt gegebene Einheit von prokreativem Tun und Transfer körperlicher Substanz aufbrach, stellte sich die Frage, aus welchem Element - Akt oder Substanz - sich Vaterschaft im Sinne von Erzeugerschaft ableitete (während die Mutterschaft weiterhin durch Empfängnis und Evidenz der Geburt bestimmt blieb). Mit anderen Worten:Problematisch wurde die Bestimmung «natürlicher» Vaterschaft ${ }^{7}$. Diese Problematisierung spielt der Faiseur d'Hommes durch. Eine Szene im Roman setzt den Dr. Knauss den Menschenmacher des Titels - in einen Dialog mit einem Dorfarzt: «Aber wie», fragt dieser, «können wir demjenigen, der als Vater gilt (père réputé), beweisen, dass er wirklich der Autor des Werkes (l'auteur de l'œuvre) ist? [...] Man könnte 〈dieses Lebenselement〉 (élément de vie), von dem Sie eben gesprochen haben, auswechseln ...» Freilich, das wäre Täuschung und Trug, wäre dunkle Machenschaft, und solches, so die Antwort des Dr. Knauss, schliesst die professionelle «Ehre» des Mediziners grundsätzlich aus ${ }^{8}$. Tatsächlich bricht die Ungewissheit von woanders her in die Köpfe und Herzen der Protagonisten ein. Das Verhältnis von Autorschaft/Vaterschaft, élément de vie/Substanz und Werk/Kind nämlich ist komplizierter, als es der Verdacht

7 Wenn hier die Problematisierung von Vaterschaft Gegenstand ist, so geht es nicht um die Doppelung von Vaterschaft als Erzeugerschaft (Vater als genitor) einerseits und sozialer und rechtlicher Vaterschaft (Vater als pater) andererseits. Vielmehr geht es allein um den Aspekt der Erzeugerschaft und damit um die Definition «natürlicher» Vaterschaft, die im hier behandelten Zeitraum ausserdem noch nicht eindeutig mit «biologischer» Vaterschaft identifiziert werden kann. Ich komme darauf zurück.

8 RamBaud/Dubut de Laforest 1884, 72. 
möglicher Vertauschung von Samen suggeriert. Die Autorschaft - so wird es die, im Sinne Zolas, zwangsläufige Ereignisfolge der fiktiven Geschehnisse im experimentellen Roman aufweisen - kommt nicht eindeutig und gemäss dem von den Autoren stark gemachten biologischen Wissen der Zeugungssubstanz, sondern gemäss traditionellen Prokreationskonzepten auch dem Zeugungsakt zu. Darin liegt die Pointe des Faiseur d'Hommes oder, anders formuliert: die «provozierte Beobachtung»" des literarischen Experiments. Gelingt im Roman das Experiment in künstlicher Befruchtung und bestätigt so die <Richtigkeit der biologischen Postulate, so ziehen die Protagonisten daraus die <falschen〉 Schlüsse hinsichtlich der Bestimmung von Vaterschaft, und die Geschichte endet in der Katastrophe.

So gelesen, gibt hier ein Werk der Fiktion aus dem 19. Jahrhundert Einblick in die komplikationsreiche Herausbildung der historisch-epistemologischen Voraussetzungen der Reproduktionsmedizin des 20. und 21. Jahrhunderts. Dazu gehörten nicht nur wissenschaftliche Theorien, medizinische Instrumente und Prozeduren. Es bedurfte auch einer neuen Deutung des prokreativen Geschehens hinsichtlich der Frage, welchen Elementen Begründungsstatus für verwandtschaftliche Zugehörigkeit und Genealogie zukommen sollte. Prokreation, so Marilyn Strathern, operiert historisch als ein «natürliches Symbol» für soziale Beziehungen und gesellschaftliche Ordnung, indem es «facts of society» in angenommenen «facts of nature» verankert. Als das aufkommende biologische Wissen diese facts of nature - und den Begriff von «Natur» überhaupt - seit dem letzten Drittel des 19. Jahrhundert neu fasste, waren davon auch die daraus abgeleiteten facts of society tangiert ${ }^{10}$. Von den Irritationen, die sich aus dieser Konstellation ergaben, handelt dieser Beitrag, und er sucht sie in der Medizin als einer Disziplin und Profession auf, in der biologisches Wissen nicht einfach angeeignet und übersetzt wird, sondern in der es auf kulturelle Deutungen stösst und dabei Widersprüche erzeugt. Insofern als der Faiseur d'Hommes genau dies dokumentiert, dient er mir hier zusammen mit medizinhistorischen Publikationen als Quelle für eine kulturhistorisch interessierte Wissenschaftsgeschichte ${ }^{11}$.

9 Zola 1881, 3 und 7.

10 Strathern 1992, 16 und 19.

11 Dieser Artikel ist Teil eines grösseren Forschungsprojekts zu Konzepten von Prokreation in den Humanwissenschaften der Moderne, das sich insbesondere mit den reproduktionsmedizinischen Experimenten im 19. Jahrhundert sowie mit der Herausbildung der pränatalen Psychologie im 20. Jahrhundert beschäftigt. 


\section{Der Menschenmacher. Ein Roman und sein Protagonist}

Welche Geschichte wird im Faiseur d'Hommes erzählt? An einem Abend im März 1867 trifft im tief im Schwarzwald gelegenen Schloss eines adligen und an seiner Kinderlosigkeit leidenden Paares ein alter Freund des Ehemannes $e^{2 i n}{ }^{12}$. Karl Knauss, der Besucher, ist ein Arzt in seinen Fünfzigern. Sein Bart ist blond und frisiert, sein Haarschopf gepflegt, seine Zähne weiss, seine Stirn ein Bild des Fleisses, der Arbeitsamkeit und der Energie. Knauss erkennt rasch die Lage der Gräfin und des Grafen von Ahlenberg und verkündet letzterem «im Namen der Wissenschaft», dass er Vater sein werde. Es folgt eine Unterrichtung über die Befruchtung von windblütigen Pflanzen und schliesslich die Analogie: «Der Pollen ist für die Blüte der Palme das, was der Keim des Lebens für den Menschen.» ${ }^{13}$ Mehr gibt der Roman nicht preis über das Verfahren der künstlichen Befruchtung, dagegen sind freilich die zeitgenössischen medizinischen Publikationen voll von technischen Details: Der Arzt lässt sich entweder vom Ehemann Sperma geben oder gewinnt dieses nach dem Akt aus der Vagina der Frau, um es dann - gleich dem Wind - in den weiblichen Unterleib einzuführen ${ }^{14}$.

Nach langwieriger Überzeugungsarbeit, die mit dem Dorfarzt, dem Pfarrer und einem Anwalt die personifizierten Instanzen gesellschaftlicher Deutungs- und Entscheidungsmacht involviert, gelingt es Knauss, Graf und Gräfin für sein Vorhaben zu gewinnen. Die künstliche Befruchtung wird vollzogen (aber nicht beschrieben), und die Gräfin flaniert bald schon schwanger und erwartungsfroh durch die Gärten des weitläufigen Anwesens. Es scheint, als könne diese Geschichte nur einen guten Ausgang haben. Stattdessen kommt es zur Komplikation: Zahlreicher werden die Spaziergänge der Gräfin, häufiger trifft sie auf Knauss und setzt sich zu ihm auf die Gartenbank. Bald darauf liegt sie fiebrig im Bett, verfällt ins Delirium, ruft Knauss an ihre Seite und entlässt ihn nicht mehr aus ihrem Blick. Kurz: Die Gräfin hat sich in den Arzt verliebt. Knauss ergeht es nicht besser. Ein Mathematiker, räsoniert er vergeblich, verliebt sich nicht in seine Formel, ein

12 Barral rechtfertigt den fiktiven Transfer des Geschehens von Frankreich, dem tatsächlichen «Zentrum der neuentstehenden Methode» (Semke 1996,37), nach Deutschland damit, dass gerade so die französische Vorreiterschaft gewürdigt werden könne; im Roman verdankt denn auch der Arzt Dr. Knauss all sein Wissen französischen Medizinern.

$13 \mathrm{RamBaud} /$ Dubut de Laforest 1884, 37.

14 In den zeitgenössischen medizinischen Texten werden insbesondere folgende Verfahrensfragen diskutiert: Ort des Eingriffs (Arztpraxis oder Wohnung der Patienten), Instrumente (Spritze oder Sonde), Injektion (intrauterin oder vaginal) und besonders der Zeitpunkt des Eingriffs. Zwar ging man allgemein davon aus, dass die Tage unmittelbar nach der Menstruation die fruchtbaren seien, aber gerade weil diesbezüglich Unsicherheiten bestanden, wurde auch empfohlen, zu verschiedenen Zeitpunkten des Zyklus zu praktizieren. 
Physiker nicht in seine Apparaturen, ein Geometer nicht in sein Messband, ein Architekt nicht in seine Entwürfe, und also: «Man verliebt sich nicht in ein wissenschaftliches Instrument.» Doch muss er sich schliesslich eingestehen, dass «die Gräfin nicht allein litt, bedrängt von diesen fast materiellen Gefühlen». Und dieses Eingeständnis der Liebe zur Frau geht einher mit einer weiteren Einsicht: «Knauss sagte sich, dass das Kind, das bald zur Welt kommen sollte, das seine sei» ${ }^{15}$.

Schliesslich gestehen sich Knauss und die Gräfin wort- und tatenlos ihre Gefühle ein, und die Gräfin bringt einen rotbackigen Sohn zur Welt. An dieser Stelle kommt es zur letzten Wendung und der Roman zu seinem Ende: Knauss verbrennt sein Manuskript mit dem Titel Die künstliche Zeugung beim Menschen und begeht Selbstmord. Er hinterlässt einen Brief an den Grafen, in welchem er dem Gelingen des «physiologischen Experiments» das Scheitern des «moralischen Experiments» gegenüberstellt und mit einem Plädoyer für die Begründung der Psychologie als einer Wissenschaft des Herzens schliesst ${ }^{16}$.

An diesem hochdramatischen Finale setzt die folgende Interpretation an - und zwar mit der Feststellung eines nicht minder dramatischen Widerspruchs: Wieso setzt ein Roman, der anwaltschaftlich für die künstliche Befruchtung eintritt, das Scheitern des sozialen und psychologischen - beziehungsweise in zeitgenössischer französischer Begrifflichkeit des moralischen - Experiments in Szene, das sich aus dem medizinischen Experiment ergibt? Anders formuliert: Wieso muss Dr. Knauss sterben?

Dass der Roman zugunsten der künstlichen Befruchtung Stellung nimmt, erschliesst sich nicht nur aus den vielen Plädoyers, die sich durch das Buch ziehen. Es erschliesst sich auch aus dem Kontext: Der Roman ist ein Schlüsselroman, steht doch die Figur des Karl Knauss für den französischen Arzt Joseph Gérard, der mit Georges Barral, dem Freund der Autoren und Verfasser des Vorworts, in enger Verbindung stand. Joseph Gérard war im Paris der 1880er Jahre als Verfechter der künstlichen Befruchtung zu einer gewissen Berühmtheit gelangt. Bereits 1877 hatte er zum Thema publiziert, und 1888 erschien ein in Zusammenarbeit mit dem Illustratoren José Roy entstandenes und an ein breites Publikum gerichtetes Ratgeberbuch ${ }^{17}$.

Diesem populärwissenschaftlichen Erfolg stand ein ramponierter akademischer Ruf gegenüber:Am 28. Juli 1885 nämlich war Gérards Thèse mit dem Titel Contribution à l'histoire de la fécondation artificielle von der Académie de Médecine unter dem Vorsitz des Gynäkologen Charles Pajot abgelehnt

$15 \mathrm{RamBaud} /$ Dubut de Laforest 1884, $223 \mathrm{ff}$.

$16 \mathrm{RamBaud} /$ Dubut de Laforest 1884, 308.

17 Gérard 1877; Gérard 1888. Letzterem sind die hier verwendeten Abbildungen entnommen. 
worden ${ }^{18}$. Der Vorgang blieb einem interessierten Publikum nicht verborgen. Die Revue Scientifique erklärte das Ereignis zur «Affäre»; zwar sei das eigentliche Ereignis «von geringfügiger Bedeutung», doch habe es «grosse (extra-medikale) öffentliche Erregung» geweckt und «absolut abwegige Kommentare» in der Presse veranlass ${ }^{19}$. Gérard selbst sprach in einem handschriftlichen Kommentar von einem «Skandal» ${ }^{20}$. Und Georges Barral sollte noch zehn Jahre später an den «Lärm» erinnern, den das Scheitern dieser Thèse verursacht habe ${ }^{21}$.

Gegenstand der Kontroverse waren unterschiedliche Beurteilungen der Beweggründe für die Ablehnung. Man sage, schreibt Gérard, die medizinische Fakultät habe «nicht öffentlich eine Theorie absegnen wollen - deren Gültigkeit sie nicht bestreiten konnte -, weil damit die Wissenschaft und die Moral den Abenteuern des Charlatanismus und der Libertinage ausgeliefert» worden wären. Solchen Sensibilitäten hätte er sich nicht verschlossen, so Gérard, doch habe Pajot selbst darauf beharrt, dass weder die «Immoralität des Gegenstandes, noch die revolutionären Konsequenzen einer solchen Thèse», sondern «schlicht und einfach eine Frage der Zahlen» die Fakultät zur Ablehnung bewogen habe ${ }^{22}$. Die Revue Scientifique schreibt im Sinne Pajots, es habe, anders als in der Presse kolportiert, «die Frage der Moral» keine Rolle gespielt; es seien schlicht die Fakten, auf die der Kandidat sich berufen habe, wissenschaftlich ungenügend erhärtet - «et voilà tout» ${ }^{23}$.

Die in der Tat bemerkenswerte Statistik im Schlussteil von Gérards Thèse führt 518 Fälle von sterilen Ehepaaren auf, die der Autor zwischen 1875 und 1885 behandelt haben will. In 52 Fällen hätten herkömmliche Behandlungsmethoden zum Erfolg geführt, in 102 Fällen sei die künstliche Befruchtung erfolgreich angewendet worden, in 41 habe diese zu Fehlgeburten geführt und in 323 Fällen schliesslich sei aus medizinischen Gründen keine Behandlung möglich gewesen, eine solche abgebrochen worden oder erfolglos geblieben. Einem Charles Pajot dürfte nicht nur eine Erfolgsquote von einem Fünftel suspekt gewesen sein, sondern auch die breite Anwendung der Praxis, hatte er doch 1882 in einem gynäkologischen Manual festgehalten, dass

18 Im Oktober desselben Jahres gelangte Gérard mit einer Thèse zu krampfadrigen Geschwüren doch noch zu Doktorwürden (vgl. Gérard 1885b).

19 Anonym 1885, 254.

20 Gérard o.J.

21 Barral 1895, 356. Für eine zusätzlich dramatische Koloratur der Ereignisse sorgte retrospektiv Barrals Bericht darüber, wie er im Winter 1885 das «einzige Exemplar, das der zerstörerischen Wut des doktoralen Aeropags der Ecole de médecine de Paris entwischt» sei, seinerseits dem Herdfeuer im Gérardschen Haushalt entrissen habe, um es im von ihm herausgegebenen Journal-Barral zu publizieren (Barral 1898, 70).

22 Gérard o.J.

23 Anonym 1885, 254. 
die künstliche Befruchtung nur in einem von hundert Fällen angezeigt sei; entsprechend instruierte er seine Studenten: Der Arzt, der die Ursachen der Unfruchtbarkeit aufzuklären wisse, so heisst es in einer Vorlesung von 1886, bedürfe «fast nie dieser Hilfe [der künstlichen Befruchtung], die einmal, rein zufällig, nützlich sein kann, wenn eine lange Serie möglicher natürlicher Prozeduren ausgeschöpft ist $»^{24}$. Auf diese Kritik an seiner Statistik reagierte Gérard mit Spott: Nichts als verletzte Eitelkeit stecke hinter der Zurückweisung seiner Thèse, gingen doch in seiner Praxis weit mehr Patientinnen ein und aus als bei den akademischen Koryphäen - was aber deshalb nicht verwundere, weil seine Türen auch «bescheidenen Leuten» offenstünden ${ }^{25}$.

Dass Gérard die Auseinandersetzung in diesen Begriffen interpretiert, dürfte auch daher rühren, dass er nicht nur ein wissenschaftlicher Aussenseiter mit abenteuerlichem Lebenslauf, sondern auch ein sozialer Aufsteiger war. Als Kind mittelloser Eltern am 17. März 1834 in Pont-à-Mousson geboren, macht Gérard gemäss autobiographischer Darstellung neunjährig eine Schreiner-, zwölfjährig eine Mechanikerlehre, erfindet 16jährig eine Dreschmaschine und meldet sich 1851 zum Militärdienst. Er kämpft als Unteroffizier im Krimkrieg, in Solferino und Sedan, steigt zum Lieutenant auf und erhält schliesslich, kriegsverletzt, die Ehrenlegionsmedaille. 1871 heiratet Gérard, holt die gymnasiale Ausbildung nach, studiert Medizin in Paris, wird 1874 Officier de santé und schifft sich als Bordarzt auf Transatlantikschiffen ein. 1875 erlangt er den Doktortitel an der University of Livingstone in den USA und lässt sich anschliessend wieder in Paris nieder, wo er ein Doktoratsstudium aufnimmt ${ }^{26} .1877$ erscheint Gérard als Médecin-directeur de la maison de santé de Bois-de-Colombes, einem Vorort von Paris ${ }^{27}$. Der Guide Rosenwald führt von 1888 bis 1898 die Praxis eines Dr. Gérard im Neunten Arrondissement auf; hier scheint er bis zu seinem Tod als Spezialist für Neurosen und Gynäkologie praktiziert zu haben.

In einem gewissen Sinn verkörpert Gérards Lebenslauf mit seiner gewundenen medizinischen Ausbildung inklusive US-amerikanischer Schlaufe das, was Charles Pajot - Professor an der Medizinischen Fakultät in Paris und als Herausgeber der Annales de gynécologie et obstétrique ein Doyen der französischen Gynäkologie - an der künstlichen Befruchtung suspekt war und ihn die Geschichte dieser Praxis in einem historisch nicht korrekten Licht

24 Pajot 1882, 665; Pajot 1886, 29.

25 Gérard o.J.

26 Diese Informationen entstammen einer autobiographischen Notiz, die dem Abdruck von Gérards Thèse im Journal-Barral vorangestellt ist (Gérard 1885a). Die Bibliothèque Nationale führt Gérard als Mediziner in Paris und ehemaligen Kavallerieoffizier. Vgl. ausserdem zu Gérard: Anonym 1877; Anonym 1898; Barral 1885b.

27 Gérard 1877. 
sehen liess. Pajot nämlich hatte sich bereits 1867 zur Thematik geäussert und die künstliche Befruchtung zu einer «Exzentrizität aus der Neuen Welt» erklärt, die sich unter der bodenständigen französischen Ärzteschaft kaum einbürgern werde ${ }^{28}$. Zwar kam der Gynäkologe James Marion Sims, der die Praxis 1866 international bekannt machte, tatsächlich aus den USA ${ }^{29}$. Doch zur selben Zeit erschien auch in Paris eine Broschüre zur künstlichen Befruchtung ${ }^{30}$. Bereits 1847, 1852, 1861 und 1864 waren bei der Académie des Sciences vier «plis cachetés» zum Thema hinterlegt worden ${ }^{31}$. Und seit mehr als sechzig Jahren existierte in Paris eine Broschüre mit dem Titel: «Anwendung der von Spallanzani an einigen Tieren vollzogenen Experimente (expériences) zur künstlichen Keimbefruchtung auf die menschliche Gattung. Oder: Ergebnis eines Experimentes, das beweist, dass man Kinder unter Mitwirkung (concours) der beiden Geschlechter, aber ohne deren Verkehr (approche) machen kann».

\section{Experimente mit künstlicher Befruchtung im Frankreich des 19. Jahrhunderts}

Mit dieser Schrift von Michel-Augustin Thouret, französischem Arzt und Direktor der Ecole de santé de Paris, hebt 1803 die dokumentierte Geschichte der künstlichen Befruchtung in Frankreich an ${ }^{32}$. Thouret bezog sich auf das Experiment, das dem italienischen Priester und Naturforscher Lazzaro Spallanzani 1780 gelungen war, als er eine Hündin mittels der Einspritzung von Samenflüssigkeit befruchtet hatte. Allerdings tätigte Thouret das davon inspirierte Experiment am Menschen nicht selbst, sondern leitete einen Ehemann dazu an. Denn obschon «rein mechanisch» (purement mécanique), komme die Prozedur nicht ohne gewisse «Präliminarien» aus, argumentiert Thouret. Diese Präliminarien bestanden im wesentlichen aus «wolllüstigen Liebkosungen», die zum Zweck hatten, das «Delirium des Sentiments» hervorzurufen ${ }^{33}$. Auf diese Gesten und Empfindungen des Begehrens konnte so

28 Pajot 1867, 217.

29 Sims' On Uterine Surgery (1866) war zuvor (1864 bis 1865) in serialisierter Form in The Lancet erschienen.

30 Dehaut 1865.

31 David 1987. Die Eingabe eines «pli cacheté» (versiegelten Dokuments) bei der Académie des Sciences bot Wissenschaftlern die Möglichkeit, eine Entdeckung oder Erfindung als die ihre zu registrieren, über die sie zum gegebenen Zeitpunkt noch nicht publizieren wollten. Die Öffnung eines pli cacheté kann nur vom Autor, seinen Erben oder aber, nach Ablauf einer Frist von 100 Jahren, von der Académie selbst veranlasst werden.

32 Die Schrift erschien anonym, wird aber übereinstimmend Thouret zugeschrieben.

33 Thouret 1803,13-16. 
lange nicht verzichtet werden, wie angenommen wurde, dass die Empfängnis das Lustempfinden der Frau voraussetzt.

Mit dieser Vorstellung bricht gut sechzig Jahre später Félix Dehaut; «sexuelle Sensibilität» sei nicht notwendig zur Empfängnis, hält er in seiner Abhandlung zur künstlichen Befruchtung fest, und damit kann deren Vollzug in die Hände des Arztes gelegt werden ${ }^{34}$. Aus der Perspektive nachfolgender Autoren, die Dehaut gern als erste französische Erörterung zum Thema handeln, beginnt erst damit die Geschichte der künstlichen Befruchtung beim Menschen ${ }^{35}$. Zwei Jahre später öffnet die Réforme médicale ihre Seiten zwei Berichten über erfolgreiche künstliche Befruchtungen ${ }^{36} .1868$ berichtet ein Dr. Girault vor der Société médicale du Panthéon über zehn Fälle, in denen er zwischen 1838 und 1861 die künstliche Befruchtung vorgenommen habe; dabei sei es in sieben Fällen zu Schwangerschaft und Geburt gekommen ${ }^{37}$. Ein Jahr später legt Jules Gautier eine an ein breites Publikum adressierte Schrift über die Praxis vor ${ }^{38}$. 1871, gut anderthalb Jahrzehnte vor Joseph Gérard, verteidigt Fabien Gigon vor einem ebenfalls von Charles Pajot präsidierten Dissertationskomitee erfolgreich eine Thèse zum Thema ${ }^{39} .1877$ äussert sich Joseph Gérard zum ersten Mal zur künstlichen Befruchtung und zielt bereits hier auf eine «Vulgarisierung» der Praxis ${ }^{40}$. 1879 berichtet Oscar Lajartre - weit spektakulärer als Gérard sechs Jahre später - von 567 Befruchtungen, die er in seiner Praxis durchgeführt habe, wovon 501 von Erfolg gekrönt gewesen seien ${ }^{41} .1883$ verhandelt die Société de médecine légale anlässlich eines Gerichtsprozesses in Bordeaux die künstliche Befruchtung und einigt sich dabei auf die Formel, dass «ein ehrenhafter Arzt» in dieser Sache nicht von sich aus die Initiative ergreifen solle, dass er aber die «Operation» auch nicht verweigern dürfe, wenn sie von den Betroffenen verlangt werde ${ }^{42} .1885$ erscheint eine Abhandlung von Louis

34 Dehaut 1865, 47.

35 So etwa bei Roubaud 1876. Roubaud erwähnt, wie in der Traditionsbildung zur künstlichen Befruchtung üblich, auch den britischen Arzt John Hunter, allerdings als nicht ernst zu nehmenden Vorläufer, da er 1776 den künstlichen Befruchtungsakt durch den Ehemann habe vornehmen lassen. Thouret wäre - hätte Roubaud ihn zur Kenntnis genommen - wohl demselben Verdikt zum Opfer gefallen. Zu Hunter vgl. Poynter 1968.

36 Gigon 1867; Lesueur 1867.

37 Girault 1868. Giraults Referat wurde 1870 auch in der Wiener Medizinischen Wochenschrift veröffentlicht.

38 Gautier 1870 [1869].

39 Gigon 1871.

40 Gérard 1877, 368.

41 Lajartre 1888 [1879].

42 Leblond 1883. Gegenstand des Prozesses waren die Honorarforderungen eines Arztes gegenüber einer Patientin; im Richterspruch wurde aber auch die künstliche Befruchtung grundsätzlich ablehnend beurteilt. 
German; 1888 schliesslich macht Gérards populärwissenschaftliches Buch zum Thema Furore ${ }^{43}$.

Wenn auch mehrheitlich von wenig bekannten Ärzten verfasst, waren all diese Abhandlungen im medizinischen Diskurs nicht marginal: Seit 1872 wurde die Thematik der künstlichen Befruchtung in gynäkologischen Handbüchern aufgegriffen, und 1878 stellte der Histologe Charles Robin das Verfahren im Dictionnaire encyclopédique des sciences médicales vor ${ }^{44}$.

Als Gérard 1885 seine Thèse verfasste und 1888 der Faiseur d'Hommes erschien, hatte die künstliche Befruchtung also als in gewissen Fällen angezeigte Sterilitätsbehandlung in die gynäkologische Theorie und Praxis Eingang gefunden - auch wenn ihr Status dort fragil, umstritten, stets legitimationsbedürftig und von einer Aura des Unbehagens umgeben war. Die Revue Scientifique notiert denn auch zur Affäre Gérard:

Was die künstliche Befruchtung betrifft, so ist sie in keiner Weise in Zweifel gezogen worden. Es handelt sich dabei um eine Operation, die ehrbare Skrupel wecken kann, die nur in Ausnahmefällen indiziert ist, die aber als eine der schönsten wissenschaftlichen Errungenschaften betrachtet werden muss. [...] heute wird sie an der Fakultät unterrichtet und in den jüngsten geburtshilflichen und gynäkologischen Abhandlungen beschrieben. ${ }^{45}$

Tatsächlich fällt Gérards Thèse nicht aus dem Rahmen der anderen Abhandlungen über die künstliche Befruchtung; sie enthält die wesentlichen Elemente, die sich in den meisten Publikationen zum Thema finden: Erklärung des Vorgangs der Befruchtung als Verschmelzung von Keimzellen, Ausführungen zu den möglichen Ursachen weiblicher und männlicher Sterilität, Bemerkungen zur Geschichte des Verfahrens, Beschreibungen der operativen Prozeduren und der verwendeten Instrumente sowie Diskussionen moralischer und gesellschaftspolitischer sowie gelegentlich auch legaler Aspekte, ausserdem persönliche Beobachtungen und allenfalls Behandlungsstatistiken.

Beim Pariser Publikum allerdings scheint Gérard in den 1880er Jahren gewiss auch als Folge seiner intensiven Popularisierungsanstrengungen zum eigentlichen Protagonisten der Praxis geworden zu sein. Der Faiseur d'Hommes trug dazu noch bei: So schreibt Gérard selbst, dieses «schöne» Buch habe ihm den Namen eines Menschenmachers eingetragen ${ }^{46}$. Allerdings dürfte ihm bei diesem Etikett nicht ganz wohl gewesen sein: «Wir machen keine Kinder à la mécanique, wie man uns vorwirft», schreibt er mit Nachdruck an anderer Stelle, «wir erschaffen keine Kinder, wir bedienen uns des

43 German 1885; Gérard 1888.

44 Courty 1872; Roubaud 1876; Leblond 1878; Eustache 1881; Lutaud 1883; de Sinéty 1892; Robin 1878.

45 Anonym 1885, 254.

46 Gérard 1888, 368. 
Vaters und der Mutter und unsere Rolle endet lange bevor die Befruchtung stattfindet $\gg^{47}$.

Dass im Roman, der den in Frankreich bekanntesten zeitgenössischen Verfechter einer der schönsten wissenschaftlichen Errungenschaften heroisiert, das Experiment der künstlichen Befruchtung in der Katastrophe der illegitimen Liebe und des Suizids endet, hängt mit dieser Aura des Unbehagens zusammen, das die Praxis umgab. Dieses Unbehagen nährte sich auch, aber nicht nur, aus moralischen Bedenken. Es dräute vor allem an der Demarkationslinie, die Gérard - ganz im Einklang mit den biologischen Erkenntnissen - zwischen dem Tun des Arztes und dem Entstehungsmoment eines neuen Wesens so entschieden zog und die aber keineswegs eindeutig war.

\section{Normale Befruchtung, Doppelpunkt. Vom Akt zur Substanz}

Die moralischen Skrupel, welche die Technik der künstlichen Befruchtung von allem Anfang an evoziert, liegen auf der Hand: Dass ein Arzt als «Dritter» in einem Akt interveniert, der an Sexuelles rührt, kann nicht unproblematisch sein und ist entsprechend begründungspflichtig. Die Rechtfertigung dazu liefert grundsätzlich der medizinische Kontext, der die Technik als bei gewissen Varianten der 〈Pathologie Unfruchtbarkeit〉 angezeigte therapeutische Intervention ausweist ${ }^{48}$. Indes ist auch in diesem Kontext Spielraum für Misstrauen, das sich nicht auf die Technologie an sich, sondern auf mögliche Missbräuche in der Anwendung richtet - namentlich der Verdacht, dass die Ärzte den Samen vertauschen und anstelle desjenigen des Ehemannes eine dritte, möglicherweise die eigene, Zeugungssubstanz einsetzen könnten.

Dieser Verdacht, der in der anfangs zitierten Romanpassage über den Nachweis der «tatsächlichen» Vaterschaft ausgesprochen wird, motiviert im Roman - in einer kruden Variante - das Brodeln der Gerüchteküche: Bei der ominösen Heldentat des Knauss handle es sich, so wollen es die bösen Stimmen im Dorf, um nichts anderes als die monumental trickreiche Finte eines gewöhnlichen Ehebrechers, der seine Schandtat unter dem Schleier eines wissenschaftlichen Experimentes zu verbergen suche. Gegen diesen Verdacht schreiben die Vertreter der künstlichen Befruchtung in ihren wissenschaftlichen Publikationen an, indem sie zum einen die Idee einer

47 Gérard 1877, 377; Hervorhebung im Original.

48 Benninghaus 2005. Nebst der therapeutischen Legitimierung finden sich in den französischen Abhandlungen auch immer wieder bevölkerungspolitische Argumente für die künstliche Befruchtung. 
heterologen Insemination gar nicht aufkommen lassen oder explizit verwerfen und indem sie zum anderen die Ehrenhaftigkeit ärztlichen Handelns betonen ${ }^{49}$. Und gegen diesen Verdacht gilt es den im fiktiven Dr. Knauss

Abb. 1. Aus: Joseph Gérard, Nouvelles causes de stérilité dans les deux sexes. Fécondation artificielle comme moyen ultime de traitement, Paris: C. Marpon \& E. Flammarion 1888 , S. 368 .
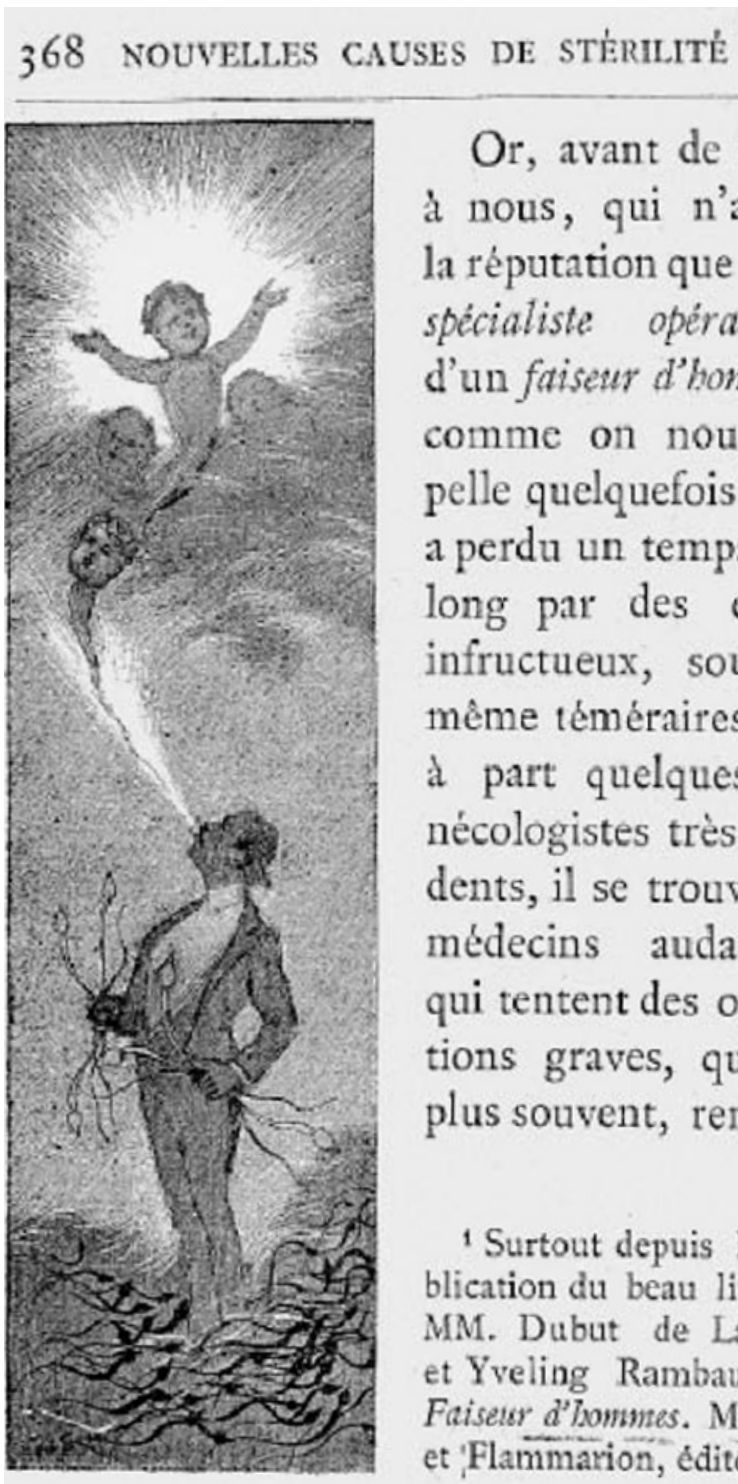

Or, avant de venir à nous, qui n'avons la réputation que d'un spécialiste opérateur, d'un faiseur d'hommes, comme on nous appelle quelquefois ', on a perdu un temps fort long par des essais infructueux, souvent même téméraires, car a part quelques gynécologistes très prudents, il se trouve des médecins audacieux qui tentent des opérations graves, qui, le plus souvent, rendent

'Surtout depuis la publication du beau livre de MM. Dubut de Laforest et Yveling Rambaud, $L$ Faiseter d'hommes. Marpon et 'Flammarion, editeurs.

49 Da die Ärzte in der Regel eine Untersuchung der Samenqualität vorsahen, war die Verwendung von Spendersamen in rein praktischer Logik auf dem Tisch. So diskutiert denn auch die Société médicale du Panthéon 1868 den Fall einer von Dr. Girault erlangten Zwillingsschwangerschaft infolge der «Substitution des Spermas eines betagten Mannes mit demjenigen eines jungen Mannes» (vgl. Girault 1869, 60). Dieser Fall wird von den Mitgliedern der Société in bezug auf mögliche rechtliche Implikationen diskutiert, aber nicht rundweg verurteilt. Die Information findet sich allerdings nicht in Giraults Fallschilderung, und in den folgenden Jahren wird die Samenspende von den Ärzten in der Regel nicht in Betracht gezogen; sie kommt aber mit Spekulationen über mögliche eugenische Anwendungen sogleich ins Spiel (vgl. z.B. Vacher de Lapouge 1896, 472). 
personifizierten künstlichen Befruchter exemplarisch reinzuwaschen. Und das geschieht raffiniert, indem die Autoren den Knauss eine moralische Transgression begehen lassen, angesichts derer er als sittlicher Heroe präsentiert werden kann: Er verliebt sich zwar in die Gräfin, räumt aber eher das Feld seiner weltlichen Existenz, als dass er sich in eine illegitime Liebesbeziehung einliesse: «Tu es père, ta femme est pure. Je vous dis adieu!», so endet sein Abschiedsbrief an den Freund und Grafen ${ }^{50}$.

Im Romanplot aber ist mehr am Werk; diese moralische Heroisierung determiniert den Selbstmord und die Vernichtung des Werks nicht: Knauss könnte - wie es als Möglichkeit angedeutet wird - den Schauplatz auch einfach verlassen, ohne sich ums Leben zu bringen und sein Opus magnum zu verbrennen. Wird dieses Motiv des Todes ernst genommen als die Negation von Existenz, die den Mediziner und sein Werk trifft, so erschliesst sich ein weiterer Interpretationsstrang: Die Ereignisfolge im Faiseur d'Hommes - so das Argument, das im folgenden entfaltet werden soll - kann gelesen werden als das Beharren auf einer vom prokreativen Akt vermittelten Einheit von Entstehung eines neuen Menschen und Elternschaft, die sich dem verweigert, was biologisches Wissen über prokreative Vorgänge nahelegt, und damit der künstlichen Befruchtung die Existenzberechtigung entzieht.

Diese Einheit stellt sich in herkömmlichen kulturellen und wissenschaftlichen Deutungen von Prokreation als ein wechselseitiger Verweisungszusammenhang dar: In der intimen körperlichen Begegnung wird ein Kind gezeugt, dessen - «natürliche» - Eltern diejenigen sind, die diesen Akt vollzogen haben. Seine Gültigkeit bezieht dieser Zusammenhang aus dem gemeinsamen Tun der prospektiven Mutter und des prospektiven Vaters. Es ist dieses gemeinsame Tun, das «Machen» von Nachwuchs als Betätigung «elterlicher Organismen $»^{51}$, das die Ärzte im letzten Drittel des 19. Jahrhunderts als akzessorisch ausweisen, ja ausweisen müssen, weil erst unter dieser Voraussetzung der Arzt den prokreativen Akt vollziehen kann.

Möglich gemacht hat diese Sicht der Dinge ein neues, auf histologischen und physiologischen Entdeckungen beruhendes Verständnis der reproduktiven Vorgänge beim Menschen, das von elterlichem Handeln abstrahierte und das prokreative Geschehen auf die Verschmelzung von Keimzellen re-

$50 \mathrm{RamBaud} /$ Dubut de Laforest 1884, 309.

51 Müller-Wille/Rheinberger 2005, 3. Von dieser Konzeption zeugt etwa die sogenannte «procréation artistique», die im 18. Jahrhundert ihren Höhepunkt erreichte. Dabei ging es darum, die Umstände des Zeugungsakts (Stellung, Mass der Erregung etc.), aber auch der Schwangerschaft und der Stillzeit hinsichtlich erwünschter Eigenschaften beim Kind zu kontrollieren. Vgl. Darmon 1981, 122ff. In transformierter Form und parallelisiert vom Diskurs über «Erbkrankheiten» und «Degeneration» tradiert die «hygienische Anleitung» in der zweiten Hälfte des 19. Jahrhunderts diese Vorstellung, vgl. Sarasin 2001, 434-441. 
duzierte. Nachdem 1824 die Penetration des Eis durch das Spermatozoid theoretisch postuliert worden war, wurde in den folgenden Jahrzehnten der jeweilige Zellkern entdeckt und die Penetration des Eies durch das Spermatozoid, die Zellfusion und schliesslich 1875/76 die Zellkernfusion beobachtet $^{52}$. Auf dieser Grundlage vermerkt der von Ärzten gern konsultierte Histologe Charles Robin 1878: «Die Befruchtung, Konzeption oder Inkarnation ist ein physiologisches Phänomen, dessen wesentliche anatomischen Agenzien das Ei einerseits und die Spermatozoen andererseits sind.» ${ }^{53}$

In diesem naturwissenschaftlich gesicherten Terrain verankern die französischen Ärzte ihre Plädoyers für die künstliche Befruchtung. Fabien Gigon zitiert den Physiologen Johannes Müller, als er festhält, dass «für die Befruchtung kein Beitrag des ganzen männlichen Organismus von Nöten ist» und «das Sperma genügt, sofern es in den Körper des Weibchens eingeführt wird». Bündig vermerkt er: «Normale Befruchtung. [...] Definition: Die Befruchtung ist das Resultat des materiellen und intimen Kontaktes einer vollständig vitalen Eizelle und einem vollständig vitalen Sperma.» ${ }^{54}$ Gleich argumentiert Joseph Gérard: «Um ein Kind zu machen, braucht es zwei Elemente: eine Zelle der Mutter, ein Spermatozoid des Vaters. Darauf beschränkt sich der Ausgangspunkt des Menschen, wie übrigens auch aller andern Lebewesen ohne eine einzige Ausnahme. $\rangle^{55}$

In diesen aus biologischem Wissen gewonnenen Definitionen für den medizinischen Gebrauch vollzieht sich eine Wendung von einem Akteurs- zu einem Substanzkonzept von Prokreation: Was einst das Tun von zwei Menschen war, wird nun zu einem physiologischen Phänomen, was die approche von Frau und Mann war, wird zum Aufeinandertreffen zweier verschieden gearteter Elemente oder Substanzen, was als anatomisches Agens eine Frau und ein Mann war, wird Ei und Spermatozoid. Mit anderen Worten: Prokreation als ein Akt, in dem Menschen, Gesten und Empfindungen zugange sind, wird zu «Befruchtung» als einem biologischen Mechanismus, bei dem Substanzen interagieren. Wer dieses Geschehen auf welche Weise in Gang setzt - Geschlechtsverkehr, Arzt - ist definitorisch unbedeutend ${ }^{56}$. Und daraus folgt: «Natürliche» Eltern sind nicht diejenigen, die tun, sondern

52 Vgl. zur Geschichte der Zeugungstheorien u.a. Cobb 2006; Jacob 2002; Pinto-Correia 1997; Farley 1992. Vgl. auch im Zusammenhang mit der künstlichen Befruchtung Schreiber 2007, 67-81.

53 Robin 1878,318

54 Gigon 1871, 16 und 10.

55 Gérard 1885a, 314.

56 Damit ist nicht gesagt, dass die Substanz zuvor keine Rolle spielte, doch wurde ihr Wirken nicht losgelöst von einer «externen Kraft» gedacht; Fortpflanzung war in diesem Sinn «Zeugung» als «eine jedesmal neue Schöpfung», bevor sie zur «Reproduktion» als «eine inhärente Eigenschaft eines jeden lebenden Systems» wurde (Jacob 2002, 25 und 27f.). 
diejenigen, welche die substanzhaften anatomischen Agenzien zu einem physiologischen Geschehen liefern ${ }^{57}$.

Das Spektakuläre und Erschütternde an der Praxis der künstlichen Befruchtung ist diese Negation der Bedeutung elterlichen Tuns: Zeugung, so beweist es das geglückte medizinische Experiment, kommt nicht nur ohne Geschlechtsverkehr aus, sie bedingt auch kein anderes gemeinsames Han-

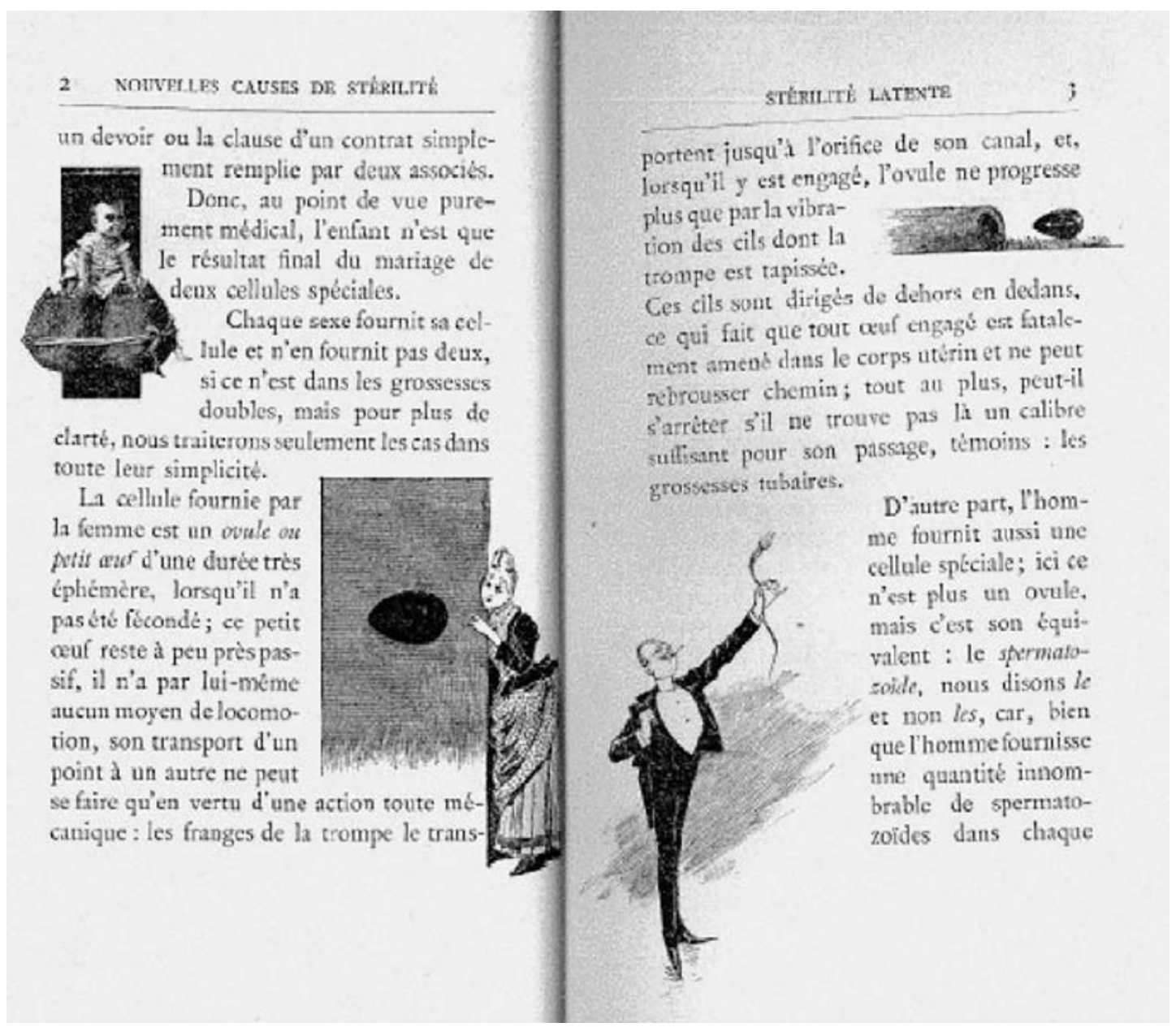

Abb. 2. Aus: Joseph Gérard, Nouvelles causes de stérilité dans les deux sexes. Fécondation artificielle comme moyen ultime de traitement, Paris: C. Marpon \& E. Flammarion 1888, S. 2 f.

57 Stellt die Biologie Wissen über die Keimzellenverschmelzung zur Verfügung, so reduziert erst die Praxis der künstlichen Befruchtung das Konzept von Prokreation auf dieses eine Element, indem sie versuchsweise den Faktor des Aktes variiert und als irrelevant auszuweisen sucht. Darin liegt der Experimentcharakter der künstlichen Befruchtung beschlossen, die das aus dem Sozialen entbettete «epistemische Ding» «Befruchtung» hervorbringt und damit ihre eigene Voraussetzung schafft. Insofern als aber - wie weiter unten gezeigt wird - die Irrelevanz des Aktes zwar postuliert, aber nicht als gewiss angenommen wird, handelt es sich bei der künstlichen Befruchtung um ein vom therapeutischen Kontext legitimiertes Menschenexperiment. Vgl. zu Experiment und epistemischen Dingen u.a.: Rheinberger 1992 und 2001. 
deln der Eltern, ja nicht einmal deren simultane Anwesenheit. Wies Thouret 1803 noch die approche - den Geschlechtsverkehr - als verzichtbar aus, nicht aber den concours - das elterliche Zusammenwirken als relevantes in Empfindungen gehülltes Tun -, so können die Ärzte nun auch darauf verzichten. Wie der Herausgeber der Dictionnaire encyclopédique des sciences médicales Amédée Dechambre räsonniert - und mit ihm Dr. Knauss im Faiseur d'Hommes:

Wenn der Blumenpollen, von den Lüften übermittelt, über grosse Distanzen hinweg Leben säen kann, wenn bei den Tieren allein die Imprägnation des Eis durch die befruchtende Substanz genügt, um die Entwicklung des Embryos ohne die aktive Kollaboration der Eltern auszulösen, wieso sollte die Frau nicht künstlich befruchtet werden können? ${ }^{58}$

\section{Triumph der Natur über die Physiologie. Irritierte Vaterschaft}

An diesem konzeptuellen Wendepunkt entsteht die Irritation, die der Roman in der Verkettung der Ereignisse dokumentiert und die Selbstmord und Werkvernichtung des Dr. Knauss motiviert. Denn die Geschehnisse verweigern sich dem biologischen Wissen dort, wo aus einem Substanzkonzept von Prokreation eine von Substanzen gestiftete Vaterschaft abgeleitet wird - mit anderen Worten: Die Geschehnisse verweigern sich einer Bestimmung von «natürlicher» Vaterschaft als «biologischer» Vaterschaft. Gewissermassen gegen diese Ableitung ordnet der Gang der Ereignisse das Arrangement der beteiligten Personen um: Bestand das Elternpaar vor dem Zeugungsakt und besteht es aus der Sicht des biologischen Prokreationskonzepts - aus der Gräfin (die empfängt) und dem Grafen (der die Zeugungssubstanz liefert), so schafft der Zeugungsakt ein zweites Elternpaar in der Formation Gräfin (die empfängt) und Arzt (der den prokreativen Akt vollzieht). Im Roman begründet weiterhin der Zeugungsakt Elternschaft - auf Umwegen, wenn es sein muss, und das heisst in diesem Fall: wenn nicht als Voraussetzung, so als Folge des prokreativen Geschehens. «Ihr Bild», erklärt die schwangere Gräfin dem Arzt, «ist in meinem Geiste stets gegenwärtig und häufig fühle ich eine Empfindung in mich eindringen, die ich nicht in Worte fassen kann. [...] Wenn bald ein kleines Wesen mich anlächeln wird, werde ich es lehren, ihren Namen zu brabbeln, gerade so wie den Namen seines Vaters.» Bald werden sich die verbal unfassbaren Empfindungen in Liebeskrankheit artikulieren und bald wird das Wie des Namens des Vaters zum Namen des Vaters: «Ja», gesteht sich Dr. Knauss ein, «es war sein Kind $»^{59}$. Mit anderen Worten:

58 Dechambre 1885, 334.

59 RamBaud/Dubut de Laforest 1884, Zitate auf den S. 191, 225, 224. 
Nicht nur die Substanz stiftet im Roman Vaterschaft, sondern auch der prokreative Akt, und weil in der künstlichen Befruchtung Akt und Substanz auseinandertreten, erzeugt der Eingriff zwei Väter.

Auf einen ersten Blick könnte diese verdoppelte Vaterschaft verstanden werden als ein Nebeneinander von «natürlichem» und gesetzlichem Vater (Graf) und «geistigem» Vater (Arzt) ${ }^{60}$. Sie ist aber mehr als das. Was im Roman als Liebe die Zugehörigkeiten neu arrangiert und das dem Akt entsprechende Elternpaar generiert, ist nichts weniger als «die Natur», die im Imaginären des 19. Jahrhunderts noch mehr als ein physiologisches Phänomen ist: Nachdem Knauss und die Gräfin sich ihre Gefühle und damit auch die gemeinsame Elternschaft eingestanden haben, sich aber dazu entschliessen, kein Liebesverhältnis einzugehen, weiss der auktoriale Erzähler zu berichten: «der Experimentator hat die Natur gezähmt; ... beide verstanden sie, dass sie aus dem Kampf siegreich hervorgegangen waren ${ }^{61}$. Es ist das vom technologischen Akt verdrängte «Natürliche» (die Zeugung eines Kindes im von Frau und Mann gemeinsam vollzogenen intimen Akt), das im Roman das Geschehen antreibt, indem es als Monströses wiederkehrt (der Treubruch, die illegitime Vaterschaft): Der Arzt ebenso wie der Graf ist «natürlicher» Vater des «künstlich» gezeugten Kindes.

Es ist die Einsicht in diesen Sachverhalt, die den horrifizierten Knauss zur Vernichtung seines Werks und in den Tod treibt. Die künstliche Befruchtung hat sich als technologisch möglich, aber als sozial und psychologisch unmöglich erwiesen: «Als physiologischer Experimentator bin ich siegreich aus dem Kampf hervorgegangen», schreibt Knauss, «dem moralischen Experiment bin ich erlegen. ${ }^{62}$ Das ist es, was ich eingangs die Pointe des Romans genannt habe, die Behauptung, die sich im Gang der Geschichte Bahn bricht: Es lässt sich zwar die Prokreation vom sexuellen Akt auf einen technologischen Akt verschieben, doch der Zusammenhang von zeugendem Akt und vaterschaftlichen Bindungen lässt sich nicht auflösen - im sozialen Imaginären bleibt Prokreation ein soziales Tun von sozialer Relevanz ${ }^{63}$.

60 Wie das in ikonographischen Inszenierungen der Geburt des ersten in vitro gezeugten Kindes 1978 denn auch der Fall sein wird.

$61 \mathrm{RamBaud} /$ Dubut de Laforest 1884, 227.

62 RamBaud/Dubut de Laforest 1884, 308.

63 Diese Deutung von Prokreation tradiert denn auch das historisch bestimmende Element von Vaterschaft, war doch Vaterschaft in euroamerikanischen Gesellschaften von einem Akt oder von sozialen Gesten etabliert - sei es im Fall der «natürlichen» Vaterschaft der Sexualakt, sei es im Fall der «legalen» Vaterschaft ein Rechtsakt (Heirat, Vaterschaftsanerkennung) und sei es schliesslich im Fall der «sozialen» Vaterschaft die Fürsorge. Vgl. zur in der Evidenz der Geburt «anerkannten» Mutterschaft und zur durch soziale Akten und Gesten «konstruierten» Vaterschaft: Strathern 1992, 148, und Smart 1987. 
Diese Beharrungskraft einer traditionellen Deutung ist nicht simpler Trägheit kultureller Konzepte beim Nachvollzug wissenschaftlichen Wissens geschuldet. Vielmehr sind hier eigentliche Widerstände am Werk. Denn mit der Transformation von einem Akt- zu einem Substanzkonzept von Prokreation wird nicht nur väterliche Abkunft an Substanz delegiert. Es wird überdies ein Element männlicher Kreativität aufs Spiel gesetzt, wird doch Vaterschaft im Kontext einer patrilinearen Gesellschaft als Zeugung verstanden und damit als ein Akt der Entäusserung männlicher Schaffens- und Gestaltungskraft, als ein Werk mit Autorschaft ${ }^{64}$. Deshalb sieht sich Barral in seinem Vorwort zum Faiseur d'Hommes gezwungen, die Angst vor einem möglichen Kreativitätsverlust zu beschwichtigen: «Drückt der Vater im Fall einer mechanischen Empfängnis dem Embryo das Zeichen seiner Kreativität auf?», fragt er - und antwortet entschieden positiv ${ }^{65}$. Gérard räumt dasselbe Problem aus, indem er argumentiert, im Fall der künstlichen Befruchtung sei der Arzt «kein Mensch, sondern ein gleichgültiges Instrument», das, so lässt sich folgern, als solches gar nicht in einen Zusammenhang, der Menschen miteinander in Verbindung setzt, eindringen und damit auch keine Autorschaft beanspruchen kann. Eine technogene Zeugung ist im Sinne des Wir erschaffen keine Kinder kein kreativer Akt, und entsprechend kann der Arzt auch nicht die Position des Vaters usurpieren ${ }^{66}$. Die Romanautoren aber lassen ihren Helden an eben diesem Problem scheitern; es bedarf eines toten Knauss', der denjenigen, der die Zeugungssubstanz zur Verfügung stellt, seiner exklusiven Vaterschaft versichert, indem er sein Werk ihm als Vermächtnis übergibt: «Tu es père.» ${ }^{67}$

Diese Szene repräsentiert die gewissermassen historische Übergabe der Vaterschaft von demjenigen, der agiert, an denjenigen, der dazu die Substanz liefert - und damit die Bestimmung von «natürlicher» Vaterschaft als «biologischer» Vaterschaft. Dass diese Übergabe im sozialen Imaginären nicht problemlos gelingt, hängt auch damit zusammen, dass der prokreative Akt im medizinischen und embryologischen Diskurs in einer anderen Hinsicht

64 Vgl. hierzu u.a. Fischer-Homberger 2001.

65 Barral 1884, xix.

66 Gérard 1888, 408. Allerdings: Just die Tatsache, dass ein Arzt als Instrument das Zeugungsgeschehen in Gang setzt, spitzt die Problematik der künstlichen Befruchtung in eine andere Richtung zu. Wenn es der Akt ist, der die Autorschaft in Fragen der Zeugung zumindest mitbegründet, dann hat die Intervention eines Instruments eine monströse Implikation: dass nämlich das künstlich gezeugte Kind gar keinen Vater hat. Diese mögliche Folgerung mobilisiert in der zeitgenössischen Diskussion allerhand Fantasien über die künstliche Befruchtung als Schritt zum man-made man. Vgl. hierzu: Arni 2008.

67 RamBaud/Dubut de Laforest 1884, 309. Dass Vaterschaft und patrilineare Genealogie in einem grundsätzlichen Sinn thematisch sind, zeigt sich im Roman übrigens nicht nur in der Geburt eines Sohnes, sondern auch in der Tatsache, dass in einem gewichtigen Nebenhandlungsstrang Erbschaftskonflikte verhandelt werden. 
durchaus bedeutsam bleibt. Barrals Frage nach der männlichen Zeugungskreativität nämlich konkretisiert die umfassendere Frage nach «dem Einfluss der künstlichen Befruchtung auf Empfängnis und Zeugung» - und das ist nicht im Sinn des gelungenen Erzeugens eines embryonalen Organismus gemeint. Es ist vielmehr die Frage danach, ob und wie die Beschaffenheit des prokreativen Aktes Eigenschaften des neuen Wesens prägt. Diese Frage hält den Akt gerade auch im Diskurs der Ärzte und Verfechter der künstlichen Befruchtung gegen ihr eigenes Argument relevant.

\section{Die Schattenwürfe des «Künstlichen». Der Akt und die Heredität}

1895 berichtet die Chronique médicale in der Rubrik «Pages oubliées de littérature médicale» von einem Gespräch zwischen Alexandre Dumas fils und den Autoren des Faiseur d'Hommes. Dumas, der ursprünglich mit einem Vorwort die Patenschaft für den Roman hätte übernehmen sollen, habe RamBaud und Dubut de Laforest zwar beraten, aber dem Werk seine Zustimmung entzogen: Die Autoren, so seine Begründung, seien zum einen «nicht weit genug gegangen»; mit ihrer Entscheidung, die «Operation» nicht zu beschreiben, hätten sie den Arzt und damit die Technik dem Verdacht des Ehebruchs gewissermassen ausgeliefert. Zum anderen sei das Buch «nicht fertig»: «Sie hören mit der Geburt des Kindes auf ... Was wird aus ihm werden? Sie antworten: ¿Es wird ein Wesen wie andere auch.> Eh bien! Nein! Das Kind, das ohne das liebevolle Zusammenwirken der Gatten erzeugt wurde, wird den andern Wesen nie gleichen. ${ }^{68}$ Diese Behauptung stellt das auf die Interaktion von Substanzen reduzierte Konzept von Prokreation nicht in Abrede. Wohl lässt sich ein Kind erzeugen ohne das liebevolle $\mathrm{Zu}$ sammenwirken von Frau und Mann; indes: Was für ein Kind kommt dabei heraus? Diese bange Frage treibt nicht nur Dumas fils um.

Zwar werden die Mediziner nicht müde, die Art des Zeugungsakts als für den Erfolg einer Befruchtung irrelevant auszuweisen. In diesem Zusammenhang sind sie nicht nur darum bemüht, das hartnäckige «Vorurteil» zu widerlegen, dem zufolge eine Frau sich in einem wollüstigen Zustand befin-

68 Dumas 1895, 743f. Das Gespräch wird von einem Redaktor als Einführung zu einem anschliessend abgedruckten Brief von Dumas an Dubut de Laforest wiedergegeben; in diesem Brief schreibt Dumas: «Deshalb habe ich Ihnen auch geraten, sich dieses neuen Faktes der génération artificielle nur als Ausgangspunkt zu bedienen, um so rasch wie möglich zur Studie und Analyse des menschlichen Wesens zu gelangen, das mit diesen rein mechanischen Mitteln erlangt worden ist. Hier würde meines Erachtens Ihr Recht als Romancier wahrhaft einsetzen.» (Dumas 1895, 745) Ein Anspruch, so möchte man anfügen, der von Mary Shelley zu Beginn des Jahrhunderts eingelöst worden war. 
den müsse, um überhaupt empfangen zu können, sondern auch darum, das Ungeheuerliche einer technogenen Zeugung zu kontern: ${ }^{69}$ Da das eigentliche Zeugungsgeschehen isoliert von menschlichem Tun innerhalb des weiblichen Uterus stattfinde, bleibe das «Künstliche» an der künstlichen Befruchtung der Prokreation äusserlich. Gleichzeitig aber beschäftigen sich die Ärzte mit der Frage, ob eben diese «Künstlichkeit» sich in den Lebenskräften und Eigenschaften des Kindes abbilden könnte - sei es in wünschenswerter oder aber in verhängnisvoller Weise. So leitet ein Herausgeber der Réforme médicale 1867 die Diskussion zum Thema mit einer Spekulation ein: Es sei «wahrscheinlich», dass «bei Wesen, die in einer Art flüchtigem Delirium empfangen worden sind, das Sinnessystem über das eigentlich intellektuelle System dominiert» und entsprechend im Fall der künstlichen Befruchtung «das Umgekehrte» der Fall wäre ${ }^{70}$. Und in einem bemerkenswerten Gedankengang von Félix Dehaut zeigt sich, wie selbst ein vom Substanzkonzept informiertes Verständnis von Prokreation nicht vollständig von herkömmlichen Konzeptionen abgelöst ist, die mit dem Nicht-Substanziellen als Formkraft rechnen: Dehaut argumentiert 1865, mit der künstlichen Befruchtung könnten «perfektere Konzeptionen» erlangt werden «als die, die den Zufällen der Kopulationen ohne Umsicht geschuldet sind», da der Arzt die «einer guten Befruchtung günstigsten Umstände» wählen könnte. Diese Umstände sind zwar materieller Art, geht es doch um die Qualität der Keimzellen. Allerdings gerät diese gerade nicht in einem materialistischen Sinn in den Blick: Man kenne, argumentiert Dehaut, den «oft sehr beträchtlichen Einfluss», den «der moralische Zustand der Eltern im Moment der Prokreation» auf das «Produkt» ausübe, deshalb sei es nur «rational» und eigentlich richtiger, hypothetisch mit diesem Einfluss auch für die Zeit zu rechnen, in der Ei und Samenzelle im Körper von Frau respektive Mann reiften ${ }^{71}$.

Neben dieser gewissermassen proto-eugenischen Hoffnung, dass die technogene Erzeugung die Entstehung erwünschter Eigenschaften befördern könnte, stand die Furcht, dass die «Künstlichkeit» des Zeugungsaktes unerwünscht Krankhaftes in die Welt bringe. So wirft Barral in seinem Vorwort zum Faiseur d'Hommes die Frage auf: «Ist die künstliche Befruchtung als ganz mechanische Operation fähig, moralisch begabte Subjekte zu schaffen?» Was die «Formen» betreffe, so sei das - die Verwendung intakter «Keime» vorausgesetzt - unzweifelhaft. Doch, so räumt er ein:

69 Vgl. z.B. Gautier 1930 [1889], 57ff.; Dehaut 1865, 47.

70 Herausgeberische Vorbemerkung zu Gigon 1867, 293.

71 Dehaut 1865, 41 f. 
Was die künftigen Instinkte [beim Kind] betrifft, die Qualitäten seiner Intelligenz, die Strebungen seines Herzens, kann es passieren, dass es in seiner ganzen moralischen und intellektuellen Organisation eine Unordnung hat, die aus ihm einen Wahnsinnigen, einen Neuropathen, einen Halluzinanten machen kann. Indes: Wir wissen darüber nichts. ${ }^{72}$

Die Frage der Machbarkeit technogener Prokreation ist so von allem Anfang an gedoppelt. Entsprechend dem biologisierten Konzept von Prokreation bringen intakte Substanzen eine intakte Form hervor. Gleichzeitig aber ist ungewiss, ob und wie die kognitiv-affektive Beschaffenheit eines solchen prokreativen Aktes die intellektuelle und emotionale Beschaffenheit des Kindes prägt. In dieser Doppelung wird das Aktkonzept von Prokreation tradiert - ganz im Sinne Thourets, der 1803 vermutete, dass «das Moralische, dessen exzessiven Einfluss auf das Physische wir kennen, das Resultat der empfundenen Sensationen notwendigerweise modifiziert ${ }^{73}$. Damit halten die hier zu Wort gekommenen Ärzte an der Vorstellung fest, dass der prokreative Akt eine eigenschaftsbildende Kraft ist. Diese Kontinuität verweist auf die zeitgenössische Offenheit des Nachdenkens über die Entstehung von Eigenschaften beim Kind, denn noch gibt es kein über verschiedene biologische und medizinische Diskurse hinweg gefestigtes Konzept einer «von den Kontingenzen der Empfängnis, der Schwangerschaft, der embryonalen Entwicklung, der Niederkunft und dem Stillen» vollständig gelösten und an Substanz gebundenen «hereditären Transmission» ${ }^{74}$. Zwar bilden sich im 19. Jahrhundert analog zum Substanzkonzept von Prokreation die epistemischen Voraussetzungen für ein Substanzkonzept von Heredität heraus, das den Ursprung von Eigenschaften in der transgenerationellen Transmission von hereditärer Substanz sehen wird. Doch der $\mathrm{Zu}$ sammenhang von Befruchtung als Produktion einer teilfähigen Zelle durch Keimzellenverschmelzung und Heredität als Reproduktion der elterlichen

72 Barral 1884, xxiii.

73 Thouret 1803,13f.

74 Müller-Wille/Rheinberger 2007,3. Die Wendung zu einem an Substanz gebundenen Konzept von «Heredität» als Ursprung von Eigenschaften wird in der Forschung unterschiedlich datiert. Die Zeitspanne, innerhalb derer sie von verschiedenen Autoren veranschlagt wird, reicht vom Ende des 18. Jahrhunderts bis zum Beginn des 20. Jahrhunderts (Müller-Wille/ Rheinberger 2007,4). Müller-Wille/Rheinberger konzeptualisieren denn auch die Emergenz von «Heredität» als die Herausbildung eines «epistemischen Raums», der sich in einem langfristigen und nicht von scharfen Brüchen, sondern von stufenweisen Verschiebungen charakterisierten Prozess entfaltet $(2005,7)$. Zentrale wissenschaftshistorische «Ereignisse» sind für den hier interessierenden Zeitraum insbesondere die 1876 erschienene Publikation der «Theory of Heredity» von Francis Galton, die eine der Transmission von Eigenschaften zugrundeliegende materiale Struktur theoretisch postuliert (Müller-Wille/Rheinberger 2005, 6f.), sowie die Erforschung des Zellkerns als eines materialen Trägers hereditärer Transmission in den 1880er Jahren (Churchill 1987). Carlos López-Beltrán (2007, 105f.) identifiziert das letzte Drittel des 19. Jahrhunderts als denjenigen Zeitraum, in dem aus verschiedenen Thematisierungsfeldern verschiedene Stränge des Denkens über «Heredität» zusammenfliessen und das «moderne Hereditätskonzept» Form annimmt. 
Qualitäten via die Zellen wird erst im letzten Drittel des 19. Jahrhunderts gemacht ${ }^{75}$. In diesem Kontext macht es durchaus Sinn, dass Ärzte und Kommentatoren über die Möglichkeit spekulieren, dass die Umstände der «künstlichen» Zeugung sich in den Eigenschaften der so erzeugten Kinder abbilden ${ }^{76}$.

So wird zwar im reproduktionsmedizinischen Diskurs avant la lettre im letzten Drittel des 19. Jahrhunderts die «natürliche» Vaterschaft respektive die väterliche Erzeugerschaft vom Akt auf die Substanz verlagert, aber zugleich bleibt Prokreation ein Akt der Kreation, der über die blosse Auslösung eines physiologischen Phänomens hinausgeht und mehr als schlichtes Medium der Transmission von Erbsubstanz ist. Damit treten unter den Bedingungen des technologischen Eingriffs in das prokreative Geschehen väterliche Erzeugerschaft und männliche Zeugungskreativität auseinander.

\section{Schlussfolgerungen}

Im «Kunsthandwerk der Repräsentation», schreibt Barbara Naumann, «erforscht die Literatur die Wissenschaft» ${ }^{77}$. Die einer solchen «forschenden» Literatur eigene Form des Experiments ist das Gedankenexperiment, das sich die menschlichen Verwicklungen des wissenschaftlichen Experiments vorstellt, den Stoff dazu aus dem sozialen Imaginären gewinnt und sich dem Risiko des ungewissen Ausgangs aussetzt. Im Versuch, so Claude Bernard, dessen experimentelle Medizin dem Zolaschen Roman expérimental Pate stand und dem Georges Barral sein Vorwort zum Faiseur d'Hommes widmete, kommen die Antworten von ausserhalb des Erwarteten ${ }^{78}$. Das Unerwartete im literarischen Versuch des Menschenmachers aber ist das Paradox, in dem sich der Experimentator verfängt: die Tatsache, dass das Kind zwei «natürliche» Väter hat, die monströse doppelte Deklaration des Dr. Knauss: Je suis père und Tu es père.

75 Duchesneau 2007.

76 Vor diesem Hintergrund wird plausibel, was der US-amerikanische Arzt David Hard von einem Fall heterologer Insemination aus dem Jahr 1886 berichtet: Der Sohn der von William Pancoast mit dem Samen seines schönsten Studenten befruchteten Frau habe, so Hard, die Gesichtszüge nicht des letzteren, sondern des «willing but impossible father»-das heisst des Ehemannes der befruchteten Frau - aufgewiesen (zit. nach Horn 1997, 147). Goethe-Leser mögen sich hier an die «Wahlverwandtschaften» erinnern, trägt doch das von Eduard und Charlotte gezeugte Kind nicht die Gesichtszüge dieser beiden, sondern des von Charlotte geliebten Hauptmannes respektive der von Eduard geliebten Ottilie.

77 Naumann 2005, 512.

78 Dagognet 1984, 18. 
Dieses Paradox ist das Symptom einer Konkurrenz der Deutungen - Akt oder Substanz -, die in traditionellen Prokreationskonzepten nicht von einander geschieden werden. Zwar bestätigt das medizinische Experiment der künstlichen Befruchtung in Roman und Arztpraxis das biologische Substanzkonzept von Prokreation; doch mündet gerade das in eine tiefgreifende Irritation und in die soziale Katastrophe. Mit dem Selbstmord des Arztes weist der Roman so die künstliche Befruchtung als vorläufig unmöglich aus, weil sie am Sinnzusammenhang scheitern muss, der im Imaginären des 19. Jahrhunderts die Intimität des Paares, die Prokreation und die Vaterschaft via den Akt in Beziehung setzt. Zugleich aber löscht der Roman die Spur dieser Unmöglichkeit in der Person des Dr. Knauss aus, um an ihre Stelle das programmatische Vermächtnis einer dereinst gelingenden Bemeisterung dieses Naturzusammenhangs zu setzen. Hat das biologische Wissen über reproduktive Vorgänge einen Anfang gesetzt, so gilt es nun, die trägen Herzen zu überzeugen. Der Fortschritt der Wissenschaft nämlich, so Dr. Knauss in seinem Abschiedsbrief, könne erst dann gelingen, «wenn die Analyse sich aller Modi der Observation bemächtigt, in Belangen der Psychologie geradesowie in denjenigen der Physiologie» ${ }^{79}$. Erst eine solche Wissenschaft und die ihr entsprechenden Techniken würden dem menschlichen Handeln die Natur vollständig verfügbar machen - die eben nicht nur im Biologischen, sondern auch im Affektiven wirkt.

Gehen wir aus einer sozialwissenschaftlichen Perspektive davon aus, dass die im Roman expérimental erforschten Köpfe und Herzen das subjektivierte Soziale sind, dann handelt der Faiseur d'Hommes nicht von uneinsichtigen Gefühlsduslern, sondern von kulturellen Konzepten, die mit biologischem Wissen im Widerstreit liegen, das seinerseits nicht frei von Widersprüchen ist. Die Irritationen, die sich mit der «Biologisierung» der Natur im Feld der Prokreation im 19. Jahrhundert auftun, sind bis heute nicht ganz verschwunden. Noch immer kreisen sie um die Frage der Bestimmung von Elternschaft ebenso wie um diejenige der Bedeutung der Art des prokreativen Aktes. Zwar hat die Unterscheidung von Akt und Substanz im Denken über Prokreation seither die Inthronisierung der Substanz als bestimmender Faktor von Zugehörigkeit möglich gemacht, die heute im Bedarf nach DNA-analytischen Vaterschaftstests für den Eigengebrauch und dem ancestry testing ebenso zum Ausdruck kommt wie in aktuellen legislatorischen Tendenzen, die Anonymität der Samenspende aufzuheben und die offene Adoption zu gewährleisten. Indes kann von einem eindeutigen Primat der Substanz nicht gesprochen werden, ist doch die genetische Abkunft gerade in diesen

79 RamBaud/Dubut de Laforest 1884, 308. 
Kontexten jeweils nur ein Aspekt unter anderen, die soziale Beziehungen und Verhältnisse begründen ${ }^{80}$. Ähnlich ambivalent verhält es sich mit der Bedeutung des prokreativen Aktes. Zwar hat sich die Reproduktionsmedizin etabliert, gleichzeitig aber ist die - nach wie vor bange - Frage, inwiefern ein technogener prokreativer Akt Eigenschaften der so erzeugten Kinder beeinflusst, heute ein Forschungsgegenstand ${ }^{81}$.

Eine experimentelle Anordnung ist dann fruchtbar, so lehren uns Wissenschaftshistoriker, wenn das Experimentieren durch «Geschehenlassen» einen «Raum für das Auftreten von unvorwegnehmbaren Ereignissen schafft ${ }^{82}$. Insofern waren die Autoren des Faiseur d'Hommes wenn auch literarisch nicht sehr begabt, so doch experimentell durchaus talentiert: Sie liessen geschehen und dabei löste sich ihr literarisches Experiment von ihrer Intention, für die künstliche Befruchtung zu plädieren. Vielleicht brachen sie deshalb das Experiment ab und schrieben nie den im Vorwort zum Faiseur d'Hommes angekündigten Folgeroman Le Fils du faiseur d'Hommes, den Alexandre Dumas fils an Stelle des Faiseur d'Hommes hätte lesen wollen ${ }^{83}$. Denn welche väterlichen Züge, so muss man sich fragen, hätte dieser Sohn im Gesicht getragen?

\section{Bibliographie}

Anonym, «Biographie Le Docteur Gérard», Revue de la Semaine 2, 8 (18 février 1877) 101-107

- «Une thèse refusée à la Faculté de médecine», Revue Scientifique 2, 8 (22 août 1885) 254

- «Les originaux de la Médecine - Le Dr Gérard et la fécondation artificielle», Chronique médicale 5, 3 (1 ${ }^{\mathrm{er}}$ février 1898) 65-71

Arni, Caroline, «Reproduktion und Genealogie. Zum Diskurs über die biologische Substanz», in: Nicolas Pethes/Silke Schicktanz (Hrsg.), Sexualität als Experiment? Identität, Lust und Reproduktion zwischen Lebenswissenschaft und Populärkultur (Frankfurt a.M./New York 2008) 293-309

Barral, Georges, «Préface», in: Yveling RamBaud/Jean-Louis Dubut de Laforest, Le Faiseur d'Hommes (Paris 1884) I-XXXI

- «Les auteurs du〈Faiseur d'Hommes»», Journal-Barral (Paris 1885a) 337-338

- «Biographie Dr Gérard», Gazette anecdotique 2 (1885b) 311ff.

80 Vgl. hierzu u.a. Büchler 2004; Freeman/Richards 2006.

81 Vgl. hierzu: Middelburg et al. 2008. Und die mit dieser Frage historisch verknüpfte Vorstellung der Formkraft der Empfindungen der empfangenden und schwangeren Frau wurde zwar seit dem 19. Jahrhundert unter dem Einfluss des modernen Hereditätskonzepts aus Medizin und Embryologie ausgelagert - aber nur, um in einer Art disziplinärer Migration die pränatale Psychologie zu begründen. Vgl. als Beispiel hierzu Khashan et al. 2008.

82 Rheinberger 2001, 82f.

83 Dem Roman Faiseur d'Hommes sollten, so Amédée Dechambre, «zwei weitere folgen: der eine handelt von der Existenz des fils du faiseur d'Hommes; im andern wird der Autor des Vorworts in Zusammenarbeit mit Yveling RamBaud und Dubut de Laforest anhand des Lebens des petit-fils die Empfängnis aus psychologischer Perspektive untersuchen» (Dechambre 1885, 334; vgl. auch Barral 1885a, 337). 
- «Les Thèses supprimées», Chronique médicale (15 juin 1895) 353-358

- [Brief an die Redaktion], Chronique médicale 5, 3 (1 ${ }^{\mathrm{er}}$ février 1898) 69-71

Benninghaus, Christa, «Eine «unästhetische Prozedur〉. Debatten über «künstliche Befruchtung» um 1910», in: Barbara Orland (Hrsg.), Artifizielle Körper-lebendige Technik. Technische Modellierungen des Körpers in historischer Perspektive, Interferenzen 8 (Zürich 2005) 107-127

Büchler, Andrea, «Sag mir, wer die Eltern sind ... Konzeptionen rechtlicher Elternschaft im Spannungsfeld genetischer Gewissheit und sozialer Geborgenheit», Aktuelle juristische Praxis AJP 10 (2004) 1175-1185

Churchill, Frederick B., "From Heredity Theory to Vererbung: the Transmission Problem, 1850-1915", Isis 78 (1987) 336-364

Cobb, Matthew, Generation. The Seventeenth-Century Scientists Who Unraveled the Secrets of Sex, Life, and Growth (New York/London 2006)

Courty, Amédée, Traité pratique des maladies de l'utérus et de ses annexes. Considérées principalement au point de vue du diagnostic et du traitement, contenant un appendice sur les maladies du vagin et de la vulve (Paris ${ }^{21872)}$

Cryle, Peter, "Love and Epistemology in French Fiction of the Fin-de-siècle", in: Dix-Neuf. Journal of the Society of Dix-Neuvièmistes 3 (2004) 56-74

Da Silva, Stella Maria, «L'imaginaire de la reproduction artificielle au XVIII ${ }^{\mathrm{e}}$ siècle - Mythomania genitalis», in: Simone Novaes (éd.), Biomédecine et devenir de la personne (Paris 1991) $89-130$

Dagognet, François, «Préface», in: Claude Bernard, Introduction à l'étude de la médecine expérimentale (Paris 1984 [1865]) 9-21

Darmon, Pierre, Le mythe de la procréation à l'âge baroque (Paris 1981)

David, Georges, «Les débuts de l'insémination artificielle au XIX ${ }^{\mathrm{e}}$ siècle: à propos de quatre plis cachetés», La Vie des Sciences. Comptes rendues de l'Académie des Sciences, Comptes rendus, série générale, tome 4, nº 5 (1987) 449-458

De Sinéty, Louis, De la Sterilité chez la Femme et de son traitement. Avec 18 figures intégrales dans le texte (Paris 1892)

Dechambre, Amédée, «Le Faiseur d'Hommes», Journal-Barral (1885) 334-337

Dehaut, Félix, De la fécondation artificielle dans l'espèce humaine comme moyen à remédier à certaines causes de stérilité chez l'homme et chez la femme (Paris 1865)

Duchesneau, François, "The Delayed Linkage of Heredity with Cell Theory”, in: Staffan MüllerWille/Hans-Jörg Rheinberger (eds), Heredity Produced. At the Crossroads of Biology, Politics, and Culture, 1500-1870 (Cambridge Mass. 2007) 293-314

Dumas, Alexandre (fils), «Dumas Alexandre (fils) et la fécondation artificielle», Chronique Médicale Scientifique, Littéraire et Anecdotique 24 (1895) 743-748

Eustache, G., Manuel pratique des maladies des femmes. Médecine et chirurgie (Paris 1881)

Farley, John, Gametes and Spores. Ideas about Sexual Reproduction, 1750-1914 (Baltimore/ London 1992)

Fischer-Homberger, Esther, "Harvey's Troubles with the Egg”, Vortrag vor der European Association for the History of Medicine and Health (Sheffield 2001)

Freeman, Tabitha/Martin Richards, "DNA Testing and Kinship: Paternity, Genealogy and the Search for the 'Truth' of our Genetic Origins", in: Fatemeh Ebtehaj/Bridget Lindley/Martin Richards (eds), Kinship Matters: Relationships and Law in a Changing Society (Oxford 2006) 67-98

Gautier, Jules, De la Fécondation artificielle dans le règne animal et de son emploi contre la stérilité (Paris 21870 [1869])

- La Fécondation artificielle et son emploi contre la stérilité chez la femme. Nouvelle édition avec figures (Paris 1930 [1889])

Gélis, Jacques, La sage-femme ou le médecin. Une nouvelle conception de la vie (Paris 1988)

Gérard, Joseph, Traité pratique des maladies de l'appareil génital de la femme, avec une notice sur la stérilité et le moyen d'y remédier par la fécondation artificielle (Paris 1877)

- De la Fécondation artificielle. Thèse refusée, o.O. o.J. (Bibliothèque Interuniversitaire de Médecine, Paris, MS 5405)

- «Contribution à l'histoire de la fécondation artificielle», in: Journal-Barral (Paris 1885a) 313-327 
- Testatheft der Académie de médecine de Paris (Paris 1885b) (Archives Nationales de France, $\mathrm{AJ} / 16 / 6864)$

- Nouvelles causes de stérilité dans les deux sexes. Fécondation artificielle comme moyen ultime de traitement (Paris 1888)

German, Louis, Fécondation artificielle de la femme (Marseille 1885)

Gigon [Art. gez. X], F., «Troisième exemple de fécondation artificielle chez la femme», Réforme médicale, 1,37 (29 septembre 1867) 293-294

Gigon, Pierre-Fabien, Essai sur la fécondation artificielle chez la femme dans certains cas de stérilité, Thèse de médicine du 28 novembre 1871, 162 (Paris 1871)

Girault, o.V., Etude sur la génération artificielle dans l'espèce humaine, lue à la Société médicale du Panthéon, Aux bureaux de l'Abeille médicale (Paris 1869) (auch publ. in: L'Abeille médicale, 25 [1868] 409-417)

Gonzalès, Jacques, Histoire naturelle et artificielle de la procréation (Paris 1996) 278-282

Griset, Nadine, L'insémination artificielle; Historique, Indications dans la stérilité du couple, unpubl. Thèse (Dijon 1995)

Hommel, Andrea, «Hermann Rohleder (1866-1934) und die Anfänge der künstlichen Befruchtung in Deutschland», Medizinhistorisches Journal 29 (1994) 121-148

Horn, David G., "Unnatural Acts: Procreation and the Genealogy of Artifice", in: Jennifer Terry/Melodie Calvert (eds), Processed Lives. Gender and Technology in Everyday Life (London/New York 1997) 145-154

Jacob, François, Die Logik des Lebenden. Eine Geschichte der Vererbung (Frankfurt a. M. 2002)

Khashan, Ali S. et al., "Higher Risk of Offspring Schizophrenia Following Antenatal Maternal Exposure to Severe Adverse Life Events", Archives of General Psychiatry 65 (2008) 146-152

Lajartre, Oscar, Des grands obstacles à la fécondation. Suppression rapide de la stérilité (Paris ${ }^{10} 1888$ [1879])

Leblond, Albert, Traité élémentaire de chirurgie gynécologique (Paris 1878)

- «Note sur la fécondation artificielle à propos d'un jugement du tribunal de Bordeaux. Rapport lu à la Société de médecine légale, Séance du 10 décembre 1883», Annales de Gynécologie (décembre 1883)

Lesueur, o.V., «Correspondance», Réforme médicale, 1, 32 (25 août 1867) 251

López-Beltrán, Carlos, “The Medical Origins of Heredity”, in: Staffan Müller-Wille/Hans-Jörg Rheinberger (eds), Heredity Produced. At the Crossroads of Biology, Politics, and Culture, 1500-1870 (Cambridge Mass. 2007) 105-132

Lutaud, Auguste Joseph, Précis des maladies des femmes (Paris 1883)

Middelburg, Karin et al., "Neuromotor, cognitive, language and behavioural outcome in children born following IVF or ICSI - a systematic review", Human Reproduction, 14,3 (2008) 219-231

Müller-Wille, Staffan/Hans-Jörg Rheinberger, "Introduction”, in: Müller-Wille/Rheinberger (eds), A Cultural History of Heredity III: 19th and Early 20th Centuries, Max-Planck-Institut für Wissenschaftsgeschichte, Preprint 294 (Berlin 2005) 3-7

- "Heredity - The Formation of an Epistemic Space", in: Müller-Wille/Rheinberger (eds), Heredity Produced. At the Crossroads of Biology, Politics, and Culture, 1500-1870 (Cambridge Mass. 2007) 3-34

Naumann,Barbara, "Introduction:Science and Literature", Science in Context 18 (2005) 511-523

Pajot, Charles, «Examen général du traité du Docteur Courty sur les affections utérines», Archives générales de médecine 9 (1867) 217

- Travaux d'obstétrique et de gynécologie. Précédés d'éléments de pratique obstétricale (Paris 1882)

- Des Obstacles à la fécondation dans l'espèce humaine. Leçon professée à la clinique d'accouchements et de gynécologie (Paris 1886)

Pinto-Correia, Clara, The Ovary of Eve. Egg and Sperm and Preformation (Chicago 1997)

Poynter, F. N. L., "Hunter, Spallanzani, and the History of Artificial Insemination", in: Lloyd G. Stevenson/Robert P. Multhauf (eds), Medicine, Science and Culture. Historical Essays in Honor of Owsei Temkin (Baltimore 1968) 97-113

RamBaud, Yveling/Jean-Louis Dubut de Laforest, Le Faiseur d'Hommes (Paris 1884)

Rheinberger, Hans-Jörg, Experiment, Differenz, Schrift. Zur Geschichte epistemischer Dinge (Marburg an der Lahn 1992) 
- Experimentalsysteme und epistemische Dinge. Eine Geschichte der Proteinsynthese im Reagenzglas (Göttingen 2001)

Robin, Charles, «Fécondation», in: Amédée Dechambre (éd.), Dictionnaire encyclopédique des sciences médicales (Paris 1864-1890), 4. Serie, Bd. 1 (1878) 318-397

Rostand, Jean, Science et génération (Paris 1948 [1940])

Roubaud, Félix, Traité de l'impuissance et de la stérilité chez l'homme et chez la femme, comprenant l'exposition des moyens recommandés pour y remédier (Paris ${ }^{3} 1876$ [1855])

Sarasin, Philipp, Reizbare Maschinen. Eine Geschichte des Körpers 1765-1914 (Frankfurt a. M. 2001)

Schreiber, Christine, Natürlich künstliche Befruchtung? Eine Geschichte der In-vitro-Fertilisation von 1878 bis 1950 (Göttingen 2007)

Semke, Iris, Künstliche Befruchtung in wissenschafts- und sozialgeschichtlicher Sicht, Marburger Schriften zur Medizingeschichte, Bd. 34 (Frankfurt a. M. 1996)

Sims, James Marion, On Uterine Surgery (New York 1866)

Smart, Carol, "There is of course the distinction dictated by nature': Law and the Problem of Paternity", in: Michelle Stanworth (ed.), Reproductive Technologies. Gender, Motherhood and Medicine (Cambridge 1987) 98-117

Stora, Danielle, Précurseurs, réalisateurs, adversaires de la fécondation artificielle humaine, unpubl. Thèse (Paris 1976)

Strathern, Marilyn, Reproducing the Future. Essays on anthropology, kinship and the new reproductive technologies (Manchester 1992)

Tanner, Jakob, «Leib-Arte-Fakt. Künstliche Körper und der technische Zugriff auf das Leben», in: Barbara Orland (Hrsg.), Artifizielle Körper - lebendige Technik. Technische Modellierungen des Körpers in historischer Perspektive, Interferenzen 8 (Zürich 2005) 43-61

Thouret, Michel-Augustin, Application sur l'espèce humaine des expériences faites par Spallanzani sur quelques animaux, relativement à la fécondation artificielle des germes, ou résultat d'une expérience qui prouve que l'on peut créer des enfants avec le concours des deux sexes mais sans leur approche (o.O. o. J. [1803])

Vacher de Lapouge, Georges, Les Sélections sociales. Cours libre de science politique (1888-1889) (Paris 1896)

Walentowitz, Saskia, «Maternités extra-ordinaires. Introduction», tsantsa. Revue de la société suisse d'ethnologie 12 (2007) 32-40

Zola, Emile, Le Roman expérimental (Paris 1881) 